\title{
O mercado de sementes e mudas de pau-rosa (Aniba spp.) no Estado do Amazonas
}

\author{
The market for seeds and seedlings of rosewood (Aniba spp.) in the State of \\ Amazonas
}

\author{
Caroline Schmaedeck Lara ${ }^{1}$ (D), Caroline Rabelo Costa $^{2}$ (D), Paulo de Tarso Barbosa Sampaio ${ }^{1}$ (D) \\ ${ }^{1}$ Instituto Nacional de Pesquisas da Amazônia, Programa de Pós-graduação em Ciências de Florestas Tropicais, Manaus (AM) \\ Brasil. E-mails: carol.slara@gmail.com; sampaio@inpa.gov.br \\ ${ }^{2}$ Instituto de Pesquisas e Educação Continuada em Economia e Gestão de Empresas, Universidade de São Paulo (USP), \\ Piracicaba (SP), Brasil. E-mail: caroline.rabelo@gmail.com
}

\begin{abstract}
Como citar: Lara, C. S., Costa, C. R., Sampaio, P. T. B. (2021). O mercado de sementes e mudas de pau-rosa (Aniba spp.) no Estado do Amazonas. Revista de Economia e Sociologia Rural, 59(3), e221035. https://doi.org/10.1590/18069479.2021 .221035
\end{abstract}

Resumo: O pau-rosa é uma das espécies com maior potencial econômico atualmente na Amazônia brasileira. Desta árvore é extraído óleo valorizado pela indústria de perfumaria, tanto que sua exploração levou a espécie à ameaça de extinção. Com a diminuição do recurso, a produção do óleo oscilou de 46 a 2 toneladas ano-1 nos últimos 20 anos, aumentando o preço de venda de USD $40.00 \mathrm{~kg}^{-1}$ a USD 320.00 $\mathrm{kg}^{-1}$ neste mesmo período. Esta valorização tem estimulado o plantio da espécie, que se depara com a limitada disponibilidade de mudas para comercialização. Diante disto, o objetivo desta pesquisa foi analisar o mercado de sementes e mudas de pau-rosa no Amazonas, identificando o número de produtores, produção e demanda média anual, preços praticados, perfil dos clientes e dificuldades na produção. Os produtores foram identificados através de pesquisas na internet e contatados para elucidar questões mais específicas da cadeia produtiva. Foi verificado que atualmente apenas três empreendedores comercializavam mudas da espécie com certa regularidade, totalizando a média de produção de 20.000 mudas/ano com preço de venda entre $R \$ 10,00$ e $R \$ 15,00$. É evidente que este mercado representa uma oportunidade promissora de negócio, que poderá obter maior êxito caso sejam estabelecidos novos arranjos produtivos.

Palavras-chave: silvicultura, Amazônia, arranjo produtivo.

\begin{abstract}
The rosewood is one of the species with the greatest economic potential in the Brazilian Amazon because its oil is highly valued by the perfumery industry. However, due to exploration the species is threatened of extinction. With the decrease in the resource, oil production has been reduced from 46 to 2 tons/year in the last 20 years, increasing the sale price from USD $40.00 \mathrm{~kg}^{-1}$ to USD 320.00 $\mathrm{kg}^{-1}$ in this same period. This valorization has stimulated the planting of the species, which is faced with limited availability of seedlings for commercialization. Therefore, his research aimed to analyze the seed and seedlings market of rosewood in Amazonas, identifying the number of producers, average annual production and demand, prices practiced, customer profile and production difficulties. Producers were identified through Internet surveys and contacted to elucidate more specific issues in the production chain. We verified that currently, only three entrepreneurs commercialize seedlings of the species with a certain regularity, totaling the average production of 20.000 seedlings/year with sale price between $R \$$ 10.00 and $R \$ 15.00$. This market represents a promising business opportunity, which can be more successful if new production arrangements are established.
\end{abstract}

Keywords: forestry, Amazon, productive arrangement. 


\section{Introdução}

O pau-rosa (Aniba spp.) é uma árvore da família Lauraceae que ocorre em toda a Amazônia Legal, incluindo o Brasil, Guiana Francesa, Suriname, Guiana, Venezuela, Peru, Colômbia e Equador (Kubtzki \& Renner, 1982). Ela iniciou a ser explorada em 1882 na Guiana Francesa devido ao óleo essencial extraído da árvore, de característico odor doce e amadeirado, muito apreciado pela indústria de perfumaria (Vial-Debas, 1996). No Brasil, destacou-se pela importância econômica que assumiu principalmente entre 1940 e 1970, quando foi produzido em larga escala, chegando a ser considerado o terceiro principal produto na balança comercial do Amazonas (May \& Barata, 2004).

Essa exploração, no entanto, foi extremamente insustentável, pois praticamente todas árvores encontradas na floresta eram derrubadas para produção de óleo nas usinas de destilação. Estima-se que entre os anos de 1937 e 2002 foram derrubadas no mínimo 825 mil árvores de pau-rosa na Amazônia brasileira, sendo exportadas aproximadamente 13 mil toneladas de óleo essencial produzidos em mais de 50 destilarias que se instalaram em municípios do interior do Amazonas e Pará (Homma, 2016). Devido à intensa exploração, atualmente é considerada ameaçada de extinção (Convention on International Trade in Endangered Species of Wild Fauna and Flora, 2011; Brasil, 1992), sendo dificilmente encontrada em populações naturais.

Desde 2011, com a inclusão da espécie no Anexo II da Convenção sobre o Comércio Internacional de Espécies da Flora e Fauna Selvagens em Perigo de Extinção (CITES), a quantidade de óleo exportada foi inferior a 225 toneladas, chegando ao valor médio de 2 toneladas ano-1 (Brasil, 2018). Esta inclusão significa que a espécie passou a fazer parte de um acordo de combate ao comércio ilegal entre os países signatários do CITES, o que, somado às restrições impostas pela legislação, coibiu a extração predatória e irregular. Por outro lado, a demanda pelo produto se manteve alta, o que levou ao aumento do preço de exportação do óleo essencial de USD $30.00 \mathrm{~kg}^{-1}$ no início da década de 1990 a aproximadamente USD 320.00 $\mathrm{kg}^{-1}$ em 2019 (Brasil, 2019).

A alta valorização do produto no mercado, somada ao incentivo promovido por Organizações Não Governamentais (ONGs) e pesquisadores, relacionado tanto à conservação quanto à possibilidade de geração de renda através da silvicultura, tem estimulado o estabelecimento de inúmeros plantios por empresários, agricultores, populações e povos tradicionais, principalmente nos Estados do Amazonas e Pará. Aliado a isto, tem sido observado que a sustentabilidade na produção pode ser alcançada através do manejo da copa em substituição ao corte raso da árvore para produção (Contim \& Contim, 2018; Krainovic et al., 2017; Sampaio et al., 2005), sendo que o óleo obtido de folhas e galhos apresenta qualidade semelhante ao da madeira (Lara et al., 2018; May \& Barata, 2004). Apesar deste contexto, são inúmeras as dificuldades encontradas pelos produtores que pretendem investir na produção deste recurso florestal, sendo a limitada disponibilidade de sementes e mudas um dos principais entraves atualmente no desenvolvimento desta cadeia produtiva.

A produção de sementes e mudas de espécies florestais é regulamentada no Brasil pelo Ministério da Agricultura, Pecuária e Abastecimento (MAPA), através da Lei $n^{\circ} 10.711$, de 5 de agosto de 2003, assim como Decretos e Instruções Normativas complementares. Esta legislação estabelece que os produtores devem se cadastrar no Sistema Nacional de Sementes e Mudas (SNSM), obtendo assim o seu Registro Nacional de Sementes e Mudas (RENASEM). Apesar de apresentar inúmeras fragilidades desde sua implementação, a legislação é importante para que produtores rurais e empresas do setor, consumidores destes produtos, tenham maior segurança em relação à origem e qualidade dos produtos adquiridos.

Devido à importância econômica da espécie e a crescente valorização de seu óleo no mercado, esta pesquisa foi realizada com o objetivo de analisar o mercado de sementes e mudas de pau-rosa no Estado do Amazonas, identificando o perfil dos produtores, os preços praticados, a produção e demanda atual, o perfil dos consumidores, a origem do material vegetativo e as principais dificuldades do setor. 


\section{Métodos}

\section{Identificação e caracterização dos produtores de sementes e mudas de pau-rosa}

Apesar de a ocorrência da espécie no Brasil já ter sido registrada nos Estados do Acre, Amapá, Amazonas, Pará e Roraima (May \& Barata, 2004; Santana et al., 1997), nesta pesquisa apenas o Estado do Amazonas foi considerado, pois é onde se concentra a produção de óleo exportada pelo país atualmente (Brasil, 2019).

Para o mapeamento dos produtores de sementes e mudas do Estado do Amazonas foi utilizado inicialmente o banco de dados do RENASEM, disponibilizado online pela Superintendência Federal de Agricultura, Pecuária e Abastecimento (SFA) do MAPA ${ }^{1}$. Com a análise dos dados disponíveis, foi possível estimar a quantidade e localização de cadastros no Estado do Amazonas, nas seguintes categorias: Comerciante, Produtor de Mudas e Produtor de Sementes. Os registros constantes com validade expirada foram desconsiderados nesta pesquisa.

Após a identificação dos cadastros, buscou-se o contato por telefone e/ou e-mail com todas as pessoas físicas e jurídicas identificadas, com o objetivo de verificar quais empreendimentos produzem atualmente a espécie alvo da pesquisa. Foi adicionada ao universo amostral uma empresa sem cadastro no RENASEM, identificada através de site de vendas da internet. Foi identificado o total de três empreendimentos que comercializavam mudas de pau-rosa com regularidade, constituindo o universo amostral desta pesquisa. Com estas informações, foi produzido mapa de distribuição dos empreendimentos utilizando o software QGIS 2.18.

\section{Análise do mercado de sementes e mudas de pau-rosa no Amazonas²}

Através do contato com os empreendedores, realizado por telefone e/ou pessoalmente, foram identificadas as seguintes questões:

Tema 1 - Produção e demanda: Qual sua produção média anual de sementes e mudas de pau-rosa (unidades)? Qual a sua produção disponível para comercialização neste momento? Qual a demanda média anual de sementes e mudas de pau-rosa que sua empresa recebe? Qual o custo de aquisição das sementes e de produção das mudas? Qual o preço de venda das mudas? A venda de pau-rosa corresponde a qual porcentagem do seu faturamento total com o negócio?

Tema 2 - Origem e perfil dos clientes: Os principais compradores são geralmente de qual município/Estado? Qual o perfil do cliente de sementes e mudas de pau-rosa (agricultores, ONGs, empresas, instituições de ensino/pesquisa, outros viveiros)? Como eles acessam seu produto? Qual a principal motivação da compra (revenda, plantio comercial, arborização, recuperação de áreas degradadas)?

Tema 3 - Origem e perfil dos fornecedores: Qual é a origem das sementes/mudas que você comercializa? Qual o perfil dos fornecedores (coletores independentes, empresas, coletadas por você ou sua empresa)? É realizada a identificação botânica das árvores? Caso seja, qual o método (exemplo: envio de ramos para herbários, checagem em livros, consulta a especialistas, funcionários do viveiro, pelo próprio coletor)?

Tema 4 - Entraves da produção: Quais são as principais dificuldades encontradas para a produção de sementes e mudas de pau-rosa?

Os dados obtidos com as entrevistas foram compilados em planilha Excel para geração dos gráficos, utilizando análises de estatística descritiva.

\footnotetext{
${ }_{1}^{1}$ Acesso disponível no site: http://sistemasweb.agricultura.gov.br/renasem/.

${ }^{2}$ Certificado de Apresentação de Apreciação Ética (CAAE): 09974519.7.0000.0006
} 


\section{Resultados e Discussão}

\section{Identificação e caracterização dos produtores de sementes e mudas de pau-rosa}

Foram identificados 18 registros no RENASEM em vigor no mês de outubro de 2018 para o Estado do Amazonas, sendo 14 destes na categoria Produtor de Mudas e apenas quatro para as categorias Produtor de Sementes (2) e Comerciantes (2). Dois destes empreendimentos possuem registro em duas categorias (Produtor de Sementes/Produtor de Mudas, Comerciante/Produtor de Mudas). Portanto, existem apenas 16 empreendimentos regulamentados atualmente no Estado junto ao MAPA, conforme a legislação específica desta atividade.

Destes, apenas três são registrados como pessoa física e dois correspondem a instituições públicas (Prefeitura Municipal e Universidade Federal), o restante (11) consiste em empresas privadas com fins lucrativos. Em relação à localização dos empreendimentos, aproximadamente $69 \%$ concentram-se em apenas dois municípios, sendo que a cidade de Maués conta com o número mais expressivo (6), seguido da capital Manaus (5). Os outros cinco municípios identificados (Apuí, Manicoré, Iranduba, Humaitá e Careiro da Várzea) possuem apenas um empreendimento regularizado no RENASEM.

O baixo número de empreendimentos regularizados no Estado pode estar associado à dificuldade dos produtores de mudas, especialmente das espécies florestais nativas, em atender às regulamentações do MAPA, resultando na atual informalidade no setor.

De acordo com Silva et al. (2015), o volume e complexidade dos documentos exigidos, o alto custo de regularização, a ausência de definição de parâmetros técnicos específicos para a maioria das espécies florestais, são alguns dos principais entraves com os quais os produtores se deparam para obter o registro do RENASEM.

Após a identificação dos empreendimentos regularizados, uma dificuldade encontrada foi a localização dos contatos dos empreendedores para avançar na coleta de dados da pesquisa. É importante ponderar que o sistema do RENASEM é bastante limitado em relação às informações disponibilizadas, que estão restritas ao período de vigência do registro e dados básicos do empreendimento (nome, localização geográfica, CPF/CNPJ). Neste sentido, a plataforma do MAPA poderia ser bastante otimizada, adicionando informações como o contato dos empreendimentos, capacidade de produção e espécies comercializadas, por exemplo.

Apenas dois empreendimentos possuem site ou rede social na internet, o que tornou necessário o esforço para localização dos telefones/emails dos produtores através do contato realizado com prefeituras, instituições de ensino e pesquisa e ONGs com atuação nos municípios de abrangência da pesquisa. Não foi possível identificar o contato de dois empreendimentos, ficando fora do universo amostral.

Dos 14 registros localizados em que o contato foi efetivado, apenas cinco têm produzido mudas de pau-rosa nos últimos anos. Nenhum destes é produtor de sementes, e dois possuem produção em pequena quantidade sem objetivos comerciais, atuando com a doação de mudas para produtores e comunidades interessadas no plantio no município de Maués. Outro empreendimento produz a espécie de forma esporádica, com produção igual ou inferior a 200 mudas $a n o^{-1}$. Estes três casos foram excluídos da pesquisa de mercado, por não apresentarem as informações requeridas de acordo com o objetivo da pesquisa. Por outro lado, foi incluído um produtor que comercializa mudas da espécie em plataforma virtual de produtos agropecuários, com o objetivo de considerar os empreendimentos com maior relevância na produção e comércio de mudas de pau-rosa atualmente no Estado, independentemente de sua regularidade junto ao MAPA.

Os resultados apresentados na Figura 1 representam os únicos três empreendimentos identificados durante esta pesquisa que comercializam com regularidade mudas de pau-rosa no Amazonas atualmente. Eles estão localizados nos municípios de Manaus, Maués e Apuí, sendo representados como Empreendimentos A, B e C, respectivamente. 


\section{Empreendimentos de produção de mudas de pau-rosa no Estado do Amazonas - 2018}
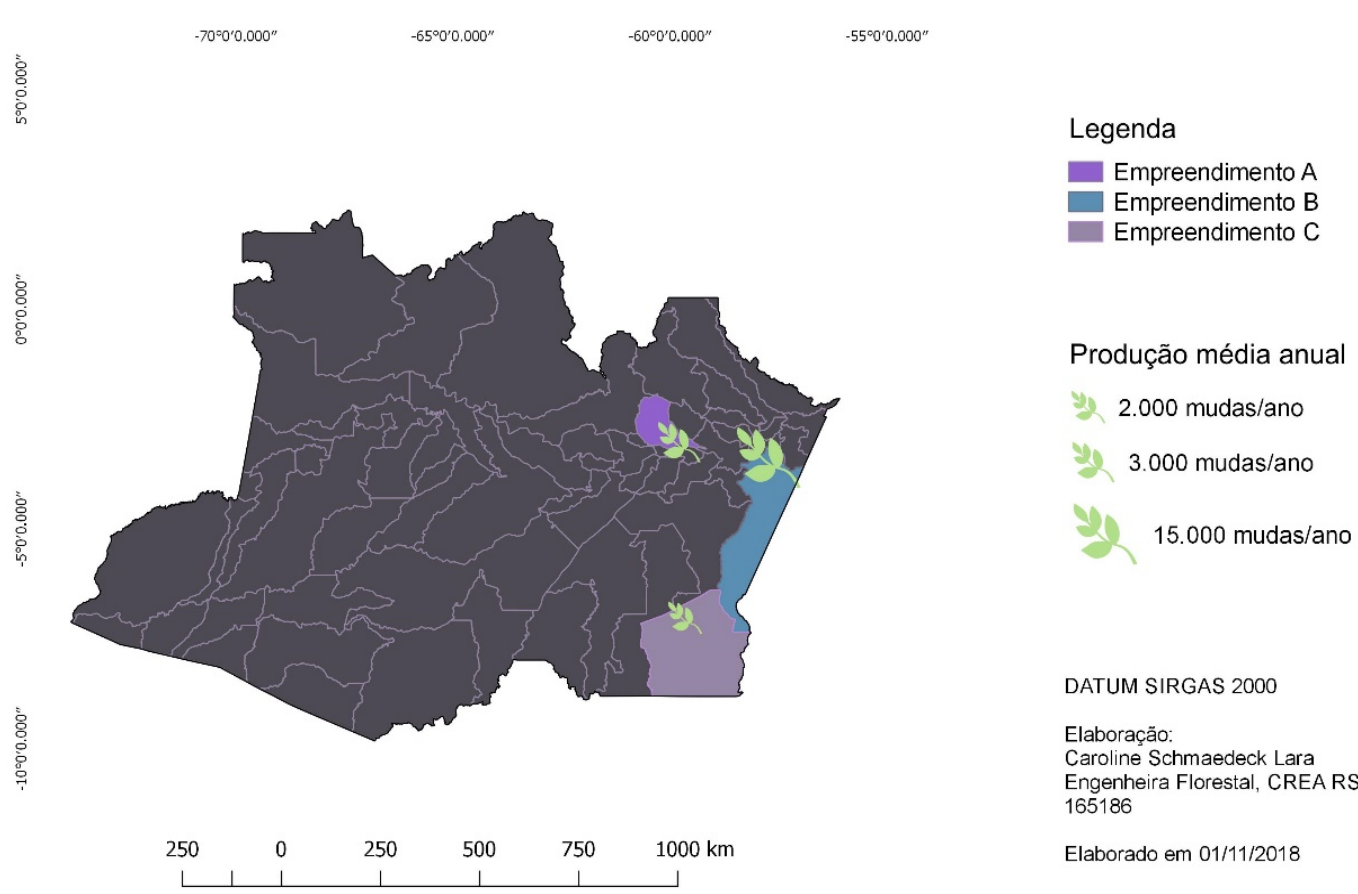

Figura 1. Localização dos empreendimentos que produzem e comercializam mudas de pau-rosa no Estado do Amazonas e produção média anual por município.

\section{Análise do mercado de sementes e mudas de pau-rosa no Amazonas}

O empreendimento $B$ foi o que apresentou maior produção média de pau-rosa, equivalente a 15.000 mudas $a_{n o}^{-1}$, seguido dos empreendimentos $A$ e $C$, com aproximadamente 3.000 e 2.000 mudas ano ${ }^{-1}$, respectivamente. Isso totaliza produção média de 20.000 mudas $a^{-1}$ para o Estado. A produção disponível para comercialização quando a pesquisa foi realizada era de 15.000, 1.000 e nenhuma muda da espécie nos empreendimentos $B, A$ e C, respectivamente. Cabe ressaltar que a frutificação da espécie é plurianual $^{3}$ (Sampaio et al., 2003) e no ano de 2018 não houve registros de produção de frutos de acordo com os entrevistados, o que restringiu o fornecimento de sementes e consequentemente a produção de mudas. A demanda média anual recebida pelos três empreendimentos foi de aproximadamente 32.000 mudas, $60 \%$ superior à produção total dos mesmos. O preço de venda praticado é igual nos empreendimentos A e B, equivalente a $R \$ 10,00$ unidade $^{-1}$, enquanto o empreendimento $C$ comercializa pelo valor de $R \$ 15,00$ unidade $^{-1}$ (Figura 2).

Todos empreendedores relataram que o preço de venda das mudas de pau-rosa é superior às demais espécies florestais nativas comercializadas por suas empresas. Esta informação corrobora o diagnóstico produzido em 2015, cujo preço médio de venda de mudas florestais verificado para a região Norte do Brasil foi equivalente a $R \$ 3,34$ unidade $^{-1}$ no varejo e $R \$ 2,23$ unidade $^{-1}$ no atacado (Silva et al., 2015). A valorização das mudas de paurosa está associada na relação oferta $x$ demanda e na dificuldade de acesso às sementes da espécie, aumentando-se assim o custo da produção, de acordo com os produtores.

${ }^{3}$ Frutificação plurianual significa que a espécie não produz frutos anualmente, seguindo um padrão irregular de reprodução. 


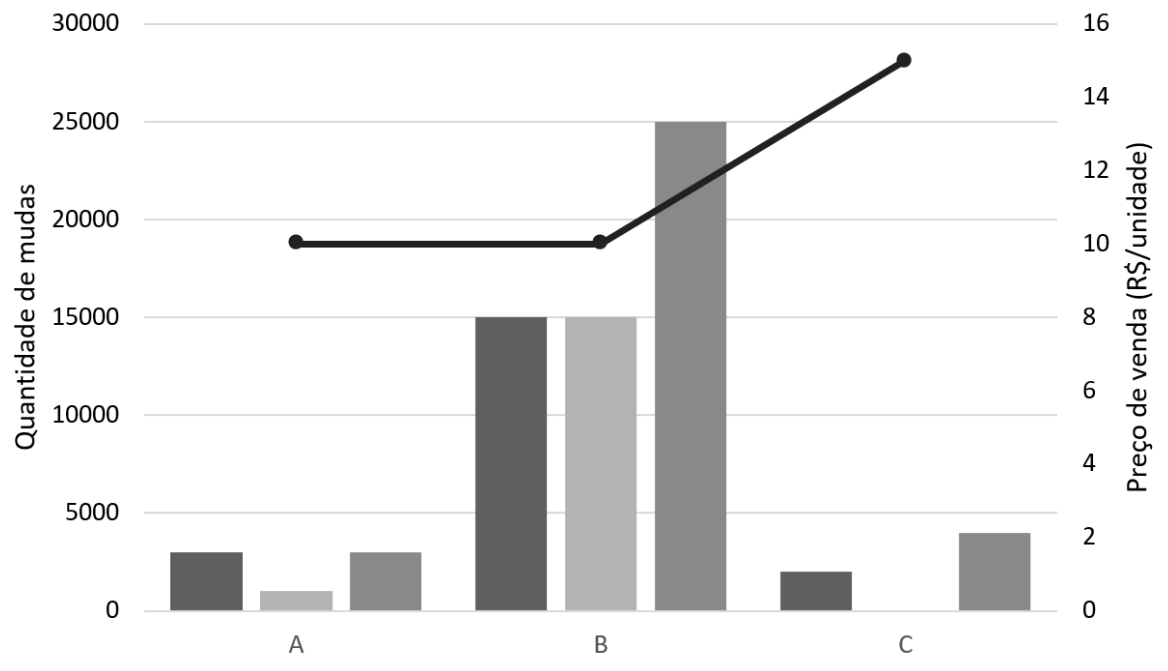

Empreendimentos

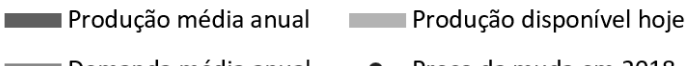

Figura 2. Informações da produção, demanda e preços de venda praticados na comercialização de mudas de pau-rosa pelos três empreendimentos.

Os três empreendimentos relataram que o faturamento relativo da venda de mudas de pau-rosa foi inferior a $10 \%$ do seu faturamento total anual. Considerando o tamanho do mercado, que de acordo com uma pesquisa de Silva et al. (2015) era de aproximadamente quatro milhões de mudas ano-1 para a região Norte do Brasil, a produção de mudas de paurosa ocupa apenas $0,5 \%$ do total comercializado. Provavelmente se a oferta de mudas da espécie fosse superior à atual, a mesma ocuparia posição de destaque no setor devido à sua rentabilidade como produto florestal.

Em relação à origem dos clientes de mudas, foram relatados empreendedores e ONGs do Amazonas, Pará e São Paulo como os principais compradores. Com menor importância, foram citados o Estado do Espírito Santo e a região Sul do Brasil. Estes clientes adquirem as mudas com fins principalmente comerciais, estabelecendo plantios para extração futura de óleo essencial ou para integrar projetos de recuperação de áreas degradadas. Em relação ao perfil e motivação de compra, os clientes são principalmente empreendedores e produtores rurais para investimento no cultivo de plantas aromáticas, assim como instituições públicas e ONGs para fomento à produção florestal sustentável, geração de renda e recuperação de áreas degradadas.

A maioria das sementes utilizadas para produção de mudas nos empreendimentos são fornecidas por coletores individuais de comunidades tradicionais e/ou indígenas, provenientes de plantios ou florestas localizadas na região dos empreendimentos. Cabe ressaltar que nenhum fornecedor de sementes de pau-rosa foi identificado durante a pesquisa, constatando que esta atividade é predominantemente realizada de forma informal. A identificação botânica das árvores utilizadas como porta-sementes é realizada na maior parte das vezes pelos próprios coletores e raramente auxiliada pela consulta a especialistas botânicos da academia.

As principais dificuldades citadas pelos produtores são a escassez de árvores matrizes na floresta nas proximidades de seus empreendimentos, a irregularidade na frutificação da espécie, além de problemas de sobrevivência das mudas devido às pragas florestais. Dessa forma, foi possível observar que o fornecimento de sementes é o principal fator limitante atualmente na cadeia produtiva estudada. Para superar esse problema, é indicado que seja fomentada a implementação de Áreas de Coletas de Sementes (ACSs) da espécie, nos municípios onde se sabe que há plantios ou árvores em populações naturais (Figura 3). 0 estabelecimento de rede colaborativa entre os diferentes atores envolvidos poderia ser 
determinante para o desenvolvimento da produção, além de promover a geração de renda para as pessoas que não possuem a intenção de investir na produzir mudas, mas têm árvores de pau-rosa em suas propriedades e que poderiam atuar na comercialização de sementes.

\section{Municípios potenciais para implementação de Áreas de Coletas de Sementes de pau-rosa no Amazonas}

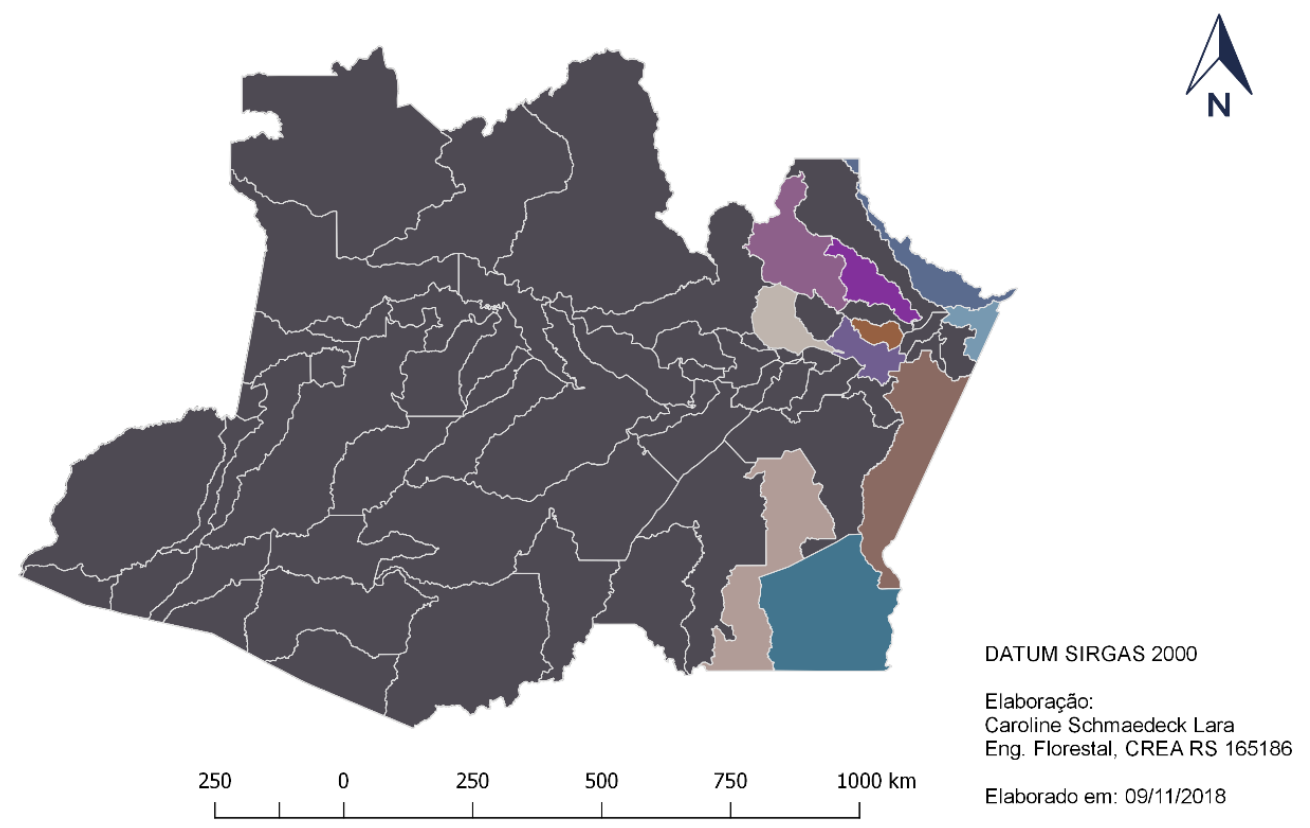

Figura 3. Municípios do Estado do Amazonas com potencial para coleta de sementes em plantios ou populações naturais de pau-rosa.

\section{Considerações finais}

O pau-rosa é espécie com potencial de geração de renda para empreendedores que desejam investir neste setor. Para viabilizar o desenvolvimento dos cultivos da espécie, é necessário superar inicialmente alguns entraves, com ações de implementação de Áreas de Coleta de Sementes para facilitar a produção de mudas; desenvolver estratégias para ampliar o apoio do poder público, universidades e ONGs para regularização dos produtores de sementes e mudas no RENASEM, além de avançar na profissionalização dos empreendimentos de produção de mudas em relação à divulgação dos produtos.

Além disso, a criação de novos modelos de negócio, considerando a necessidade de estabelecimento de arranjos produtivos com a colaboração entre produtores interessados, poderá fortalecer os empreendimentos e facilitar a aquisição de mudas para implementação de plantios pelos clientes interessados.

\section{Agradecimentos}

Agradecemos aos empreendedores que participaram da pesquisa, disponibilizando as informações solicitadas.

\section{Referências}

Brasil. Instituto Brasileiro do Meio Ambiente. (1992, abril 3). Portaria IBAMA n 37-N, de 3 de abril de 1992. Reconhece como Lista Oficial de Espécies da Flora Brasileira Ameaçadas de Extinção a relação que se apresenta. Diário Oficial [da] República Federativa do Brasil, Brasília.Brasil. Ministério do Desenvolvimento, Indústria e Comércio Exterior. (2018). Recuperado em 16 abril de 2018, de http://comexstat.mdic.gov.br/pt/home 
Brasil. Ministério do Desenvolvimento, Indústria e Comércio Exterior. (2019). Recuperado em 12 dezembro de 2019, de http://comexstat.mdic.gov.br/pt/home/

Convention on International Trade in Endangered Species of Wild Fauna and Flora - CITES. (2011, april 18-21). IFRA Position on CITES rules for Guaiacwood and Rosewood. In $19^{\circ}$ meeting of the Plants Committee. Geneva: CITES.

Contim, L. A. S., \& Contim, L. S. R. (2018). A tecnologia produtiva do pau-rosa (Aniba rosaeodora Ducke) como aliada ao desenvolvimento sustentável da região amazônica. Inclusão Social, 12(1), 199-207.

Homma, A. K. O. (2016). Políticas para expansão do plantio de pau-rosa na Amazônia. In P. T. B. Sampaio \& Z. R. C. Thomé (Eds.), Uso e Conservação do Pau-Rosa (1. ed., pp. 137-148). Manaus: EDUA.

Krainovic, P. M., Almeida, D. R. A., Desconci, D., Veiga-Júnior, V. F., \& Sampaio, P. T. B. (2017). Sequential management of commercial rosewood (Aniba rosaeodora ducke) plantations in central Amazonia: Seeking sustainable models for Essential oil production. Forests, 8(12), 438. http://dx.doi.org/10.3390/f8120438

Kubitzki, K., \& Renner, M. (1982). Flora Neotropica 1982, Monograph No. 31. New York: The New York Botanical Garden.

Lara, C. S., Barata, L. E. S., Sampaio, P. T. B., Eberlin, M. N., \& Fidelis, C. H. V. (2018). Linalool enantiomeric distribution in rosewood-reminiscent populations in Central Amazon. The Journal of Essential Oil Research, 30(6), 464-469. http://dx.doi.org/10.1080/10412905.2018.1492464

May, P. H., \& Barata, L. E. S. (2004). Rosewood Exploitation in the Brazilian Amazon: Options for Sustainable Production. Economic Botany, 58(2), 257-265. http://dx.doi.org/10.1663/0013-0001

Sampaio, P. T. B., Barbosa, A. P., Vieira, G., Spironello, W. R., \& Bruno, F. M. S. (2005). Biomassa da rebrota de copas de pau-rosa (Aniba rosaeodora Ducke) em plantios sob sombra parcial em floresta primária. Acta Amazonica, 35(4), 491-494. http://dx.doi.org/10.1590/s004459672005000400014

Sampaio, P. T. B., Ferraz, I. D. K., \& Camargo, J. L. C. (2003). Pau-rosa, Aniba roseodora Ducke. In I. D. K. Ferraz. Manual de Sementes da Amazônia (Vol. 3, 6 p.). Manaus: INPA.

Santana, A., Ohashi, S., Rosa, L., \& Green, C. L. (1997). Brazilian Rosewood Oil. The International Journal of Aromatherapy, 8(3), 16-20.

Silva, A. P. M., Marques, H. R., Luciano, M. S. F., Santos, T. V. M. N., Teixeira, A. M. C., \& Sambuichi, R. H. R. (2015). Gargalos da regulamentação da produção e comercialização de sementes e mudas florestais nativas no Brasil: contribuições para revisão da normativa. Boletim Regional, Urbano, Ambiental, 12, 71-80.

Vial-Debas, C. (1996). Da tora ao linalol: uma interface economia-ecologia. In L. Emperaire. A Floresta em jogo: o extrativismo na Amazônia Central (pp. 183-190). São Paulo, UNESP. 